\title{
Zukunftsgefährdung statt Krisenlehren - WSI-Verteilungsbericht 2010
}

\begin{abstract}
Die weltweite Finanz- und Wirtschaftskrise hat erneut gezeigt, dass die erheblich ungleichen Einkommensverteilungen sozial wie ökonomisch von Nachteil sind - nicht nur im nationalen Rahmen, sondern auch im internationalen Zusammenhang. Trotzdem betreibt die Politik ein „Weiter so“: Umverteilung von unten nach oben und Exportorientierung. Die deutsche Politik weist somit wichtige Lehren aus der Krise zurück. Schlimmer noch: Sie bereitet die nächsten Krisen mit vor. Die anhaltende Binnenmarktschwäche und soziale Polarisierung in Deutschland sind nationale Zukunftshypotheken; die steigende Belastung unserer „exportbedrohten“ Nachbarländer ist eine europäische Hypothek; die Verfestigung internationaler Handels- und Finanzierungsungleichgewichte ist ein weltweiter „eingebauter“ Destabilisator.
\end{abstract}

\section{Die gesamtwirtschaftliche Entwicklung}

2009 stieg die Lohnquote vor der öffentlichen Umverteilung - entgegen dem bis dato beobachtbaren Trend - kräftig an, weil bei krisenbedingt sinkendem Bruttoinlandsprodukt (BIP) und entsprechend rückläufigem Volkseinkommen die Gewinne in absoluten Größen einbrachen, während die absoluten Lohneinkommen wegen diverser alter und neuer Stabilisatoren (darunter die Intensivierung der Kurzarbeit, der Abbau von Arbeitszeitguthaben usw.) immerhin nur stagnierten. Die tatsächliche und die strukturbereinigte Bruttolohnquote legten im Vorjahresvergleich zu 2008 jeweils um drei Prozentpunkte zu (Tabelle 1). Doch schon wenig später im ersten Halbjahr 2010 mehrten sich die Zeichen, dass sich eine erneute Umkehr durchsetzt: jetzt wieder in die altbekannte Richtung. Beide Formen der Bruttolohnquote erreichen in den ersten Monaten 2010 bereits fast erneut das niedrige Vorkrisenniveau von 2008. Denn BIP und Volkseinkommen wachsen wieder - und darunter im ersten Halbjahr 2010 laut Statistischem Bundesamt (2010a) die Gewinneinkommen mit $19,5 \%$ „wie gewohnt" deutlich schneller als die Lohneinkommen mit 1,9\% im Vergleich zum ersten Halbjahr 2009 -, selbst wenn das zweite Halbjahr diese Schere wegen der darin gezahlten Urlaubs- und
Weihnachtsgelder noch etwas korrigieren könnte. Zwar wächst auch die Zahl der Beschäftigten. Aber der davon ausgehende „Mengeneffekt“ auf die Lohnsumme wird offenbar teilweise konterkariert durch den „Preiseffekt" bescheidener effektiver Lohnzuwächse im Allgemeinen und noch niedrigerer Lohnzuwächse im Besonderen, weil der Beschäftigungszuwachs zu einem erheblichen Teil auf mehr prekäre Jobs mit schlechter Bezahlung zurückgeht.

Noch ernüchternder ist der Blick auf die Verteilung nach der öffentlichen Umverteilung (Tabelle 2): Die Nettolohnquote hat sich 2009 in der engeren Abgrenzung (Tabelle 2, Spalte A) kaum erhöht; in der weiteren Abgrenzung (ebd., Spalte B), in der sich der Wachstumseinbruch deutlicher niederschlägt, nur um knapp eineinhalb Prozentpunkte. Und im ersten Halbjahr 2010 ist die Nettolohnquote nicht nur wieder gesunken; sie unterschreitet sogar in beiden Abgrenzungen das ohnehin schon niedrige Vorkrisenniveau mehr oder weniger deutlich, auch wenn hier im zweiten Halbjahr noch die bereits erwähnten Korrekturen nach oben kommen sollten. Fakt bleibt demnach: Die verfügbare private Nachfrage aus Lohneinkommen ist in den letzten Jahren auf nur noch gut ein Drittel der gesamtwirtschaftlichen Nachfrage gesunken und kann somit die Binnennachfrage nicht beleben.

Wichtige Hinweise zur unterschiedlichen Entwicklung von Brutto- und Nettolohnquote liefert Tabelle 3. Zwar sind 2009 und im ersten Halbjahr 2010 die relativen
Lohnsteuerlasten auf Arbeitseinkommen gesunken. So wurde seit 2009 der Eingangssteuersatz leicht (um einen Prozentpunkt) gesenkt und der Grundfreibetrag in Verbindung mit der Kindergelderhöhung deutlich angehoben. Auch haben im betrachteten Zeitraum zum einen die zahlreichen Kurzarbeiter mit vermindertem laufenden Einkommen den steuerlichen Progressionseffekt umgekehrt; zum anderen haben neue prekäre Jobs mit niedrigem Einkommen nur niedrige Steuern erzeugt. Nicht auszuschließen ist ferner, dass die Rücknahme der Restriktionen bei der steuerlichen Absetzbarkeit von Fahrtkosten zur Arbeit gleichfalls mit dazu beigetragen hat, die Lohnsteuerkosten zu senken. Jedoch: Gleichzeitig sind die Belastungen durch Sozialversicherungsbeiträge weiter gestiegen und haben die Steuerentlastungen kompensiert.

Hier überlagern sich allerdings statistisch-definitorische und politischhandlungsbedingte Veränderungseffekte. Zunächst geht die Erhöhung der Beitragslasten im Wesentlichen auf die 2009 eingeführte Krankenversicherungspflicht für alle Erwerbstätigen, auch für Selbststän-

Claus Schäfer, Dr., ist Leiter der Abteilung Wirtschafts- und Sozialwissenschaftliches Institut in der Hans-Böckler-Stiftung (WSI). Arbeitsschwerpunkt: Verteilungsanalyse von Einkommen und Lebenslagen. e-mail: claus-schaefer@boeckler.de 
Tabelle 1: Bruttolohn- und -gewinnquote - Deutschland. Das volkswirtschaftliche Einkommen aller Sektoren vor der öffentlichen Umverteilung

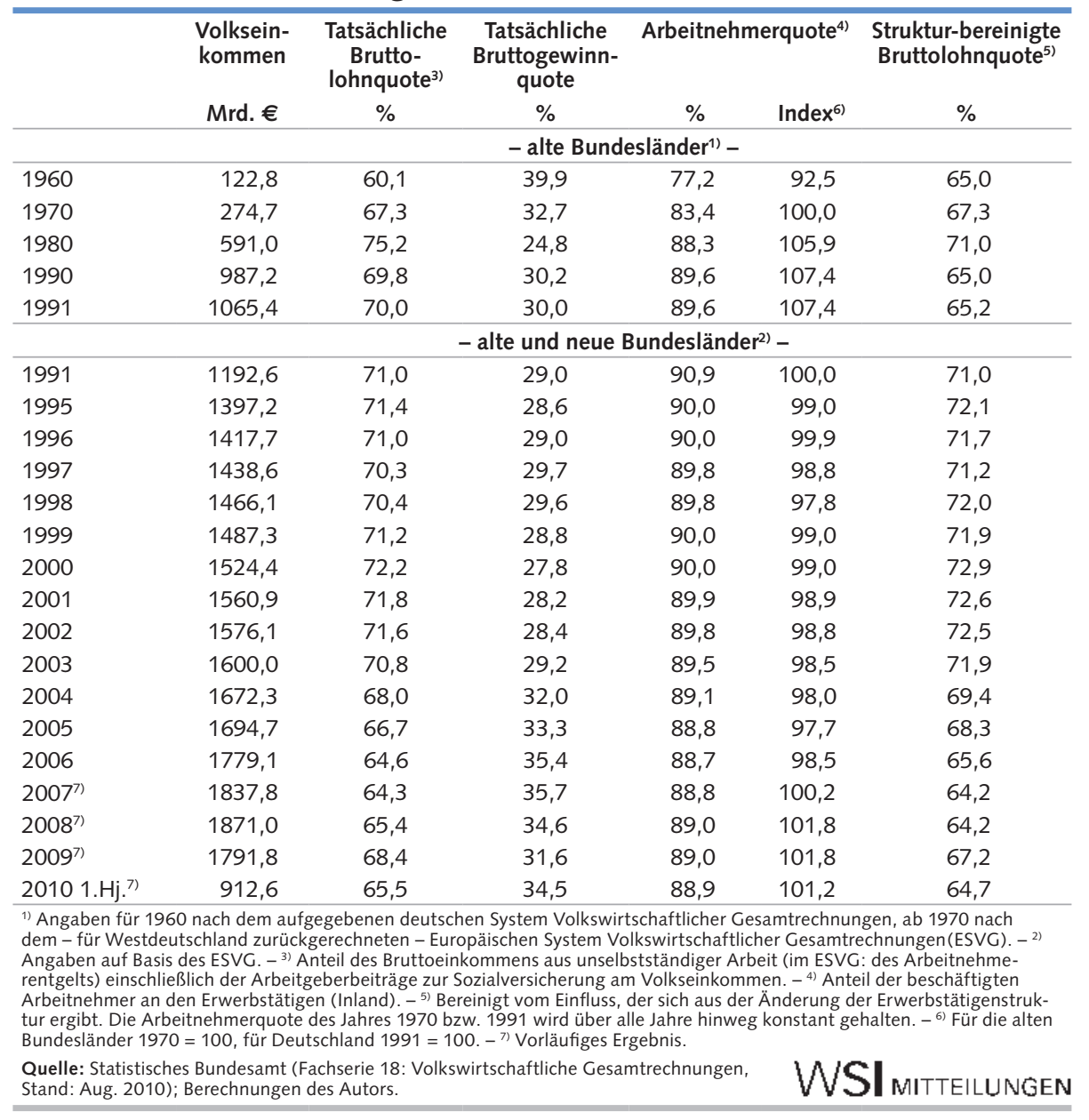

dige, zurück. Denn diese Pflicht hat das Statistische Bundesamt (2010a) veranlasst, in der Volkswirtschaftlichen Gesamtrechnung (VGR) auch die Beiträge an private Versicherungen als Sozialbeiträge zu buchen und damit rechnerisch einen Lastenanstieg auszuweisen. Der überdeckt z.B. die 2009 erfolgte Absenkung des Beitragssatzes zur Arbeitslosenversicherung von 3,3 \% auf 2,8 \%, die bis Ende 2010 befristet ist. Andererseits aber sind in den Beiträgen gemäß VGR nach wie vor nicht enthalten

- die neuen Zusatzbeiträge, die die gesetzlichen Krankenkassen (GKV) seit 2009 erheben können und teilweise von ihren Versicherten schon einfordern;

- „alte“ Zusatzlasten der Arbeitnehmer durch die Praxisgebühr;

- steigende Zuzahlungen und Aufzahlungen für Medikamente und andere Heilmittel sowie

- Eigenleistungen für nicht mehr von den Kassen erstattete Gesundheitsausgaben.
Schon 2001 wurde der entsprechende private Aufwand seitens der damaligen Bundesregierung auf rund $10 \mathrm{Mrd}$. $€$ geschätzt. Er dürfte heute wegen der zwischenzeitlichen Änderungen bei der GKV deutlich höher liegen (Gerlinger 2010). Insofern könnte der zunächst statistisch-definitorisch bedingte Anstieg der Beitragslasten den tatsächlichen Lasten durchaus entsprechen.

\section{Erwerbseinkommen im Einzelnen}

\subsection{LÖHNE}

\section{GENERELLE TRENDS}

Diese gesamtwirtschaftliche Einkommensentwicklung auf der aggregierten Ebene der VGR wird von differenzierenden anderen empirischen Quellen unterlegt. So bildet insbesondere die Verdienststatistik des Statistischen Bundesamtes (2010b) für Haupttypen von Beschäftigungsverhältnissen und deren Bezahlung die unterschiedliche Krisenbetroffenheit im Jahr 2009 relativ gut ab (Tabellen 4 und 5): In Westdeutschland sind Vollzeit-, Teilzeitund Minijobs meist abgebaut worden, Letztere vor allem in der Industrie bzw. im Produzierenden Gewerbe. In Ostdeutschland mit deutlich weniger Industriearbeitsplätzen und noch weniger exportabhängigen Betrieben ist der Rückgang nicht ganz so stark wie im Westen (bei Teilzeit hat sogar ein leichter Zuwachs stattgefunden). Mit dem Rückgang der Vollzeitarbeit aber, der in absoluten Zahlen den größten Jobverlust unter allen Beschäftigungsverhältnissen ausmacht, dürfte das schon vor der Krise im internationalen Vergleich sehr niedrige und zugleich besonders schnell gesunkene Gewicht von Vollzeitstellen am Arbeitsmarkt (Eichhorst et al. 2009) noch einmal geringer geworden sein.

Bei den verbliebenen Beschäftigungsverhältnissen ist die bezahlte durchschnittliche Arbeitszeit in Vollzeit und Teilzeit nahezu überall (mit Ausnahme der Teilzeit in den Dienstleistungen) gesunken. ${ }^{1}$ Ansonsten gehen meist verminderte Arbeitszeiten - gerade in der von der Krise besonders betroffenen Industrie - mit verminderten laufenden Verdienstzahlungen und/oder verminderten Einkünften aus Sonderzahlungen wie Urlaubs- und Weihnachtsgeld einher. Folge ist also in vielen Fällen eine verminderte Monatszahlung. Insofern darf man sich bei der Bewertung der Entwicklung in der Krise nicht auf die überall positiven Zuwächse bei den Stundenverdiensten beschränken.

\section{OST-WEST-DIFFERENZEN}

Diese Zuwächse sind in Ostdeutschland leicht höher als in Westdeutschland und bewirken 2009 - zusammen mit der geringeren Krisenbetroffenheit Ostdeutschlands - erneut ein geringfügiges Heranrücken des ostdeutschen Verdienstniveaus pro Arbeitstunde an die westdeutschen

1 Die Arbeitszeit der Minijobs wird vom Statistischen Bundesamt nicht erfasst; wahrscheinlich aber geht sie teilweise über die früher gesetzlich begrenzten 15 Wochenstunden hinaus und führt so bei den ausgewiesenen Monatseinkommen zu sehr niedrigen Stundenverdiensten. 
Verdienstverhältnisse (vgl. Tabelle 5, die letzten beiden Spalten und jeweils die dritte Unterspalte). Im gesamtwirtschaftlichen Durchschnitt (Tabelle 5, vorletzte Zeile) beträgt das Niveau im Osten 2009 ohne Sonderzahlungen $75,0 \%$ des westdeutschen (2008: 75,3\%), mit Sonderzahlungen 73,5\% (2008: 72,5\%). Damit ist die Ost-West-Kluft für ein seit 20 Jahren vereintes Land immer noch außerordentlich groß (vgl. dazu generell kritisch die Arbeitsgruppe Alternative Wirtschaftspolitik 2010a).

\section{DIFFERENZEN ZWISCHEN INDUSTRIEN UND DIENSTLEISTUNGEN}

Neben den anhaltenden Ost-West- wie auch Geschlechter-Unterschieden bei der Bezahlung verdienen mehr als bisher ebenfalls die Verdienstdifferenzen zwischen Industrien und Dienstleistungen Aufmerksamkeit. Hierzu zeigt eine tiefer gehende aktuelle Untersuchung im Auftrag des Instituts für Makroökonomie und Konjunkturforschung (IMK) zu den Lohnund sonstigen Arbeitskosten durch Ludwig (2010), dass insbesondere die im europäischen Vergleich sehr „billigen“ unternehmensnahen bzw. zuliefernden Dienstleistungen in Deutschland wesentlich zur starken und gestiegenen preislichen Wettbewerbsfähigkeit der Exportwirtschaft beigetragen haben. In dieses Bild fügt sich der Befund von Eichhorst et al. (2010) ein, wonach die in Deutschland im internationalen Vergleich überdurchschnittlich stark vermehrte prekäre Beschäftigung mit niedriger Bezahlung vor allem im Dienstleistungsbereich verbreitet ist.

\section{DIFFERENZEN NACH ALTER, QUALI- FIKATION UND BESCHÄFTIGUNGS- SEGMENT}

Offenbar zeichnet sich Deutschland im internationalen Vergleich noch durch eine vierte Disparität bei den Arbeitseinkommen aus. Laut Forschungsnetzwerk „Wage Indicator" (2010) - an dem das WSI mit der „Lohnspiegel“-Befragung von deutschen Beschäftigten teilnimmt - hat sich sowohl der Lohnabstand von qualifizierten und ungelernten Beschäftigen als auch der zwischen Berufseinsteigern bis 31 Jahre und der nächsten älteren Beschäftigtengruppe von 32 bis 40 Jahren deutlich erhöht; dagegen ist dieser Lohnabstand in anderen westeuropäischen Ländern gesunken. Alle

Tabelle 2: Nettolohn- und -gewinnquote - Deutschland 1)2) Einkommen der privaten Haushalte nach öffentlicher Umverteilung - in \% -3)4)

\begin{tabular}{|c|c|c|c|c|c|c|}
\hline \multirow[t]{2}{*}{ Jahr } & \multicolumn{2}{|c|}{ Netto-Lohnquote ${ }^{5)}$} & \multicolumn{2}{|c|}{$\begin{array}{l}\text { Nettoquote monetäre } \\
\text { Sozialleistungen }{ }^{6}\end{array}$} & \multicolumn{2}{|c|}{ Netto-Gewinnquote ${ }^{7)}$} \\
\hline & $A^{3)}$ & $B^{4)}$ & $A^{3)}$ & $\mathrm{B}^{4)}$ & $A^{3)}$ & $\mathrm{B}^{4)}$ \\
\hline \multicolumn{7}{|c|}{ - alte Bundesländer -1) } \\
\hline 1960 & 55,8 & 43,7 & 20,9 & 16,4 & 24,4 & 19,1 \\
\hline 1970 & 55,7 & 44,4 & 21,2 & 16,9 & 25,9 & 20,6 \\
\hline 1980 & 52,7 & 43,8 & 26,2 & 21,8 & 24,3 & 20,2 \\
\hline 1990 & 48,5 & 38,5 & 24,8 & 19,7 & 29,7 & 23,6 \\
\hline \multicolumn{7}{|c|}{ - alte und neue Bundesländer -2) } \\
\hline 1991 & 48,1 & 40,3 & 21,8 & 18,3 & 29,8 & 25,0 \\
\hline 1995 & 44,5 & 37,9 & 23,8 & 20,2 & 31,2 & 26,6 \\
\hline 1996 & 43,4 & 37,1 & 25,3 & 21,6 & 30,9 & 26,4 \\
\hline 1997 & 42,0 & 36,0 & 25,4 & 21,8 & 32,1 & 27,5 \\
\hline 1998 & 42,3 & 36,1 & 25,5 & 21,8 & 31,8 & 27,2 \\
\hline 1999 & 42,6 & 36,8 & 25,7 & 22,2 & 31,2 & 27,0 \\
\hline 2000 & 43,1 & 37,4 & 25,7 & 22,3 & 30,8 & 26,7 \\
\hline 2001 & 42,9 & 37,8 & 25,8 & 22,7 & 31,0 & 27,3 \\
\hline 2002 & 42,7 & 37,6 & 26,5 & 23,3 & 30,4 & 26,7 \\
\hline 2003 & 41,6 & 36,8 & 26,8 & 23,6 & 31,2 & 27,5 \\
\hline 2004 & 42,0 & 36,1 & 26,3 & 22,6 & 31,3 & 26,8 \\
\hline 2005 & 41,2 & 35,5 & 25,9 & 22,3 & 32,6 & 28,1 \\
\hline 2006 & 40,5 & 34,0 & 25,3 & 21,3 & 33,8 & 28,5 \\
\hline $2007^{8)}$ & 41,0 & 33,9 & 24,5 & 20,3 & 34,1 & 28,2 \\
\hline $2008^{8)}$ & 40,9 & 34,3 & 23,8 & 20,0 & 34,9 & 29,3 \\
\hline 20098) & 41,1 & 35,7 & 25,9 & 22,5 & 32,6 & 28,3 \\
\hline 2010 1.Hj. ${ }^{8)}$ & 39,4 & 34,0 & 26,2 & 22,6 & 34,0 & 29,3 \\
\hline \multicolumn{7}{|c|}{$\begin{array}{l}\text { 1) Bis 1990: Angaben nach dem aufgegebenen System der deutschen Volkswirtschaftlichen Gesamtrechnungen (dVGR).- } \\
\text { 2) Ab 1991: Angaben auf Basis des Europäischen Systems Volkswirtschaftlicher Gesamtrechnungen (ESVG). - 3) In \% des } \\
\text { verfügbaren Einkommens aller privaten Haushalte nach der öffentlichen Umverteilung (Spalte A). Die drei Quoten der pri- } \\
\text { vaten Haushalte addieren sich nicht genau zu } 100 \% \text { wegen statistischer Abgrenzungsprobleme. - 4) In \% des Volkseinkom- } \\
\text { mens aller Sektoren vor der öffentlichen Umverteilung gemäß Tabelle } 1 \text { (Spalte B). Die drei Einkommensquoten der privaten } \\
\text { Haushalte stellen zusammen zwar den Großteil des gesamtwirtschaftlichen Volkseinkommens; daneben sind aber auch noch } \\
\text { die Einkommen von Unternehmen und Staat zu berücksichtigen. - 5) Nach Abzug von Lohnsteuer und Sozialabgaben. - } \\
\text { 6) Im ESVG: Empfangene monetäre Sozialleistungen und übrige laufende Transfers von Staat und anderen Sektoren (ohne } \\
\text { Schadensversicherungsleistungen) abzüglich: Lohnsteuer auf Pensionen, Sozialbeiträge des Staates für Empfänger sozialer } \\
\text { Leistungen, Eigenbeiträge der Empfänger sozialer Leistungen, verbrauchsnahe Steuern und Verwaltungsgebühren. - 7) Diese } \\
\text { Kategorie wird vom Statistischen Bundesamt nicht explizit ausgewiesen: Betriebsüberschuss/Selbstständigeneinkommen plus } \\
\text { saldierte Vermögenseinkommen, abzüglich: direkte Steuern sowie Soziallbeiträge auf private Einkommen aus Unternehmertä- } \\
\text { tigkeit und Vermögen (direkte Steuern: veranlagte Einkommensteuer, nicht veranlagte Steuer vom Ertrag, Vermögensteuer). } \\
\text { - 8) Vorläufiges Ergebnis. }\end{array}$} \\
\hline \multicolumn{4}{|c|}{$\begin{array}{l}\text { Quelle: Statistisches Bundesamt (Fachserie 18, Stand: Aug. 2010); } \\
\text { Berechnungen des Autors. }\end{array}$} & & & ILUNC \\
\hline
\end{tabular}

diese Disparitäten kumulieren in mehr prekären Jobs; schlimmer noch: in einem wachsenden Niedriglohnsektor, der ja schon lange auch niedrig bezahlte Vollzeit umfasst (dazu generell Castel/Dörre 2009).

Gemessen an der international gebräuchlichen Niedriglohnschwelle von zwei Dritteln des Median-Stundenlohns waren laut Institut für Arbeit und Qualifikation (IAQ) in Deutschland zuletzt (2008) 18,4 \% aller Beschäftigten oder 6,55 Mio. Personen im Niedriglohnsektor angesiedelt: knapp $13 \%$ aller Vollzeitbeschäftigen, $25 \%$ der sozialversicherungspflichtigen Teilzeitbeschäftigten, fast $40 \%$ der befristet Beschäftigten und sogar $86 \%$ der Minijobber (Kalina/Weinkopf 2010). Bemerkenswert ist dabei nicht nur die gestiegene Zahl der Niedriglohnempfänger; mindestens ebenso problematisch ist nach IAQ ihr gleichzeitig gesunkenes Durch- schnittseinkommen. Es findet eine Armutsintensivierung in der sich ausbreitenden Armut trotz Arbeit statt. Nun wurde und wird diese Entwicklung immer wieder damit gerechtfertigt, dass durch niedrige Löhne (und Lohnkosten) zusätzliche Beschäftigung erzeugt und nicht etwa "teure“ Vollzeitarbeit verdrängt werde. Doch dafür liefert gerade der deutsche Arbeitsmarkt mit Blick auf den letzten Aufschwung laut Garz (2010) keinen Beleg.

Die Beschäftigungsentwicklung 2010 knüpft bruchlos an die Muster der Vergangenheit an, weil selbst der sozialversicherungspflichtige Zuwachs überwiegend schlecht bezahlte Leiharbeit, Teilzeitbeschäftigung und befristete Beschäftigung betrifft. Der Zuwachs der Arbeitskosten von z.B. 0,4 \% im 2. Quartal (Statistisches Bundesamt 2010c) spiegelt diese Entwicklung wider. Und auch der Anstieg der 


\begin{tabular}{|c|c|c|c|c|}
\hline \multicolumn{5}{|c|}{$\begin{array}{l}\text { Tabelle 3: Abgabenbelastung von A } \\
\text { privaten Haushalte - Deutschland } \\
- \text { in } \%-1)\end{array}$} \\
\hline \multirow[t]{3}{*}{ Jahr } & $\begin{array}{l}\text { Lohnsteuer- } \\
\text { belastung der } \\
\text { Bruttolöhne } \\
\text { und -gehälter }\end{array}$ & $\begin{array}{l}\text { Belastungen durch } \\
\text { Sozialbeiträge auf } \\
\text { Bruttolöhne und } \\
\text {-gehälter }\end{array}$ & $\begin{array}{l}\text { Belastung durch } \\
\text { direkte Steuern'2) auf } \\
\text { Gewinn- und } \\
\text { Vermögenseinkommen }\end{array}$ & $\begin{array}{l}\text { Belastung durch } \\
\text { Sozialbeiträge }{ }^{3)} \text { auf } \\
\text { Gewinn- und } \\
\text { Vermögenseinkommen }\end{array}$ \\
\hline & $\%$ & $\%$ & $\%$ & $\%$ \\
\hline & \multicolumn{4}{|c|}{ - alte Bundesländer - } \\
\hline 1960 & 6,3 & 9,4 & 20,0 & 3,0 \\
\hline 1970 & 11,8 & 10,7 & 16,1 & 2,9 \\
\hline 1980 & 15,8 & 12,8 & 15,3 & 3,9 \\
\hline \multirow[t]{2}{*}{1990} & 16,2 & 14,2 & 9,8 & 3,0 \\
\hline & \multicolumn{4}{|c|}{ - alte und neue Bundesländer - } \\
\hline 1991 & 16,3 & 14,3 & 8,1 & 3,1 \\
\hline 1995 & 18,7 & 15,6 & 4,9 & 3,5 \\
\hline 1996 & 19,3 & 16,0 & 4,2 & 4,0 \\
\hline 1997 & 19,5 & 16,8 & 3,4 & 3,7 \\
\hline 1998 & 19,5 & 16,6 & 4,7 & 3,6 \\
\hline 1999 & 19,6 & 16,4 & 6,2 & 3,5 \\
\hline 2000 & 19,4 & 16,1 & 7,7 & 3,5 \\
\hline 2001 & 18,6 & 16,0 & 7,7 & 3,4 \\
\hline 2002 & 18,7 & 16,1 & 6,7 & 3,4 \\
\hline 2003 & 18,7 & 16,4 & 5,6 & 3,2 \\
\hline 2004 & 17,5 & 16,5 & 5,4 & 3,2 \\
\hline 2005 & 17,2 & 16,7 & 5,9 & 2,9 \\
\hline 2006 & 17,5 & 17,2 & 7,1 & 2,8 \\
\hline $2007^{4)}$ & 17,9 & 17,1 & 8,3 & 2,7 \\
\hline $2008^{4)}$ & 18,2 & 17,3 & 8,5 & 2,5 \\
\hline $2009^{4)}$ & 17,8 & 17,8 & 8,8 & 3,5 \\
\hline $20101 \mathrm{Hj}^{4)}$ & 17,3 & 18,2 & 8,8 & 3,4 \\
\hline \multicolumn{5}{|c|}{$\begin{array}{l}\text { 1) In \% der jeweiligen Bruttoeinkommen. }{ }^{2)} \text { Veranlagte Einkommensteuer; nicht veranlagte Steuer vom Ertrag; Vermögen- } \\
\text { steuer. - }{ }^{\text {3) }} \text { Pflicht- und freiwillige Beiträge. - }{ }^{4)} \text { Vorläufiges Ergebnis. }\end{array}$} \\
\hline $\begin{array}{l}\text { Quelle: Statist } \\
\text { Berechnungen }\end{array}$ & $\begin{array}{l}\text { Bundesamt }(F \\
\text { Autors. }\end{array}$ & e 18, Stand: Aug. 20 & & VSI MITTEILUNGEN \\
\hline
\end{tabular}

nominalen Stundenlöhne von Vollzeitbeschäftigten im 2. Quartal 2010 um 3,4 \% widerspricht der Beschäftigungsentwicklung nur scheinbar. Denn dieser Anstieg ist verzerrt bzw. überhöht durch drei Faktoren:

- einen statistischen Basiseffekt, weil im Bezugsquartal 2009 die Verdienste noch um $12 \%$ gesunken sind;

- einen Arbeitszeiteffekt, weil im Vergleich zu 2009 jetzt Kurzarbeit spürbar abnimmt und sogar Mehrarbeit teilweise wieder zunimmt;

- weil im Bereich „Versicherungs- und Finanzdienstleister" die dortigen 850.000 Beschäftigten im Durchschnitt 7,4 \% mehr erhalten (Statistisches Bundesamt 2010d). ${ }^{2}$

Innerhalb der insgesamt schlecht bezahlten Dienstleistungsbereiche gibt es also zumindest diese „gute“ Ausnahme der Finanzdienstbranche und ihrer Angestellten. Diese Branche und ihre Besonderheit lenkt den Blick - auch wegen der von ihr mit zu verantwortenden Krise-zu den Gewinnen im eigentlichen Sinn. durchschnittlich 2,3\% gestiegen sind. Damit wird für die erste Führungsebene unter der jeweiligen Unternehmensspitze ein durchschnittliches Jahresniveau von $132.000 €$ erreicht, für die zweite Ebene immerhin noch $97.000 €$ inklusive jeweils „leistungsabhängiger“ Bestandteile.

$\mathrm{Zu}$ den Jahresgehältern der Unternehmensvorstände im engeren Sinn (aber ohne Vorstandsvorsitzende) ist z.B. aus einer Studie über die Verhältnisse in börsennotierten Unternehmen zu erfahren, dass sie 2009 als Folge der Finanzkrise mit durchschnittlich $751.000 €$ immerhin um $15 \%$ niedriger lagen als auf dem aufschwungbedingten Verdienstgipfel 2007 (Kuhner et al. 2010). Der Anteil derjenigen, die aktienkursabhängig bezahlt werden, hat 2009 mit 7 \% (vgl. ebd.) zwar unter dem mehrjährigen Mittel gelegen, werde aber generell in der Öffentlichkeit überschätzt. Auch daraus ziehen Kuhner et al. den Schluss, dass die Gehälter von Führungskräften noch immer zu wenig auf negative Unternehmensentwicklungen reagierten. Das gilt offenbar besonders für Bankmanager unter ihren großen öffentlichen Schutzschirmen. So sind zwar die Vorstandsgehälter bisher in den vom Staat direkt gestützten Kreditinstituten auf $\max .500 .000 €$ pro Jahr begrenzt worden, die Gehälter der Führungsebene darunter wurden allerdings nicht limitiert. Berechtigtes Unverständnis erregen auch die 1.400 Mitarbeiter der HRE-Bank, die gerade zusammen 25 Mio. $€$ Sonderzahlungen für 2009 erhalten haben, obwohl ihre Bank in jenem Jahr 2,2 Mrd. - vom Staat ausgeglichenen - Verlust machte (Süddeutsche Zeitung 2010a).

\section{GEWINNE VON BANKEN}

Gesamtwirtschaftlich und gesellschaftspolitisch viel relevanter sind natürlich die Gewinne der Unternehmen selbst. Hervorzuheben ist zunächst die Gewinnentwicklung der Banken seit Krisenbeginn. So schreibt die Deutsche Bundesbank: „Der aggregierte Bilanzverlust der deutschen Kreditwirtschaft hat sich 2009 gegenüber 2008 zurückgreifen muss, deren methodischer Hintergrund bzw. deren Zuverlässigkeit nur selten transparent ist. So geht aus einer Befragung der Managementberatung Kienbaum (2010) bei 7.300 Unternehmen und darin mehr als 6.000 erfassten Personen hervor, dass die Gehälter der „leitenden Angestellten“ unterhalb der Geschäftsführung 2010 gegenüber dem Vorjahr um
2 Allerdings ist auch dieser Durchschnitt irreführend, weil - wie die Frankfurter Rundschau (2010a) berichtet - der kräftige Verdienstzuwachs auf die rund 100.000 leitenden Bankangestellten in der sogenannten Leistungsgruppe 1 zurückgeht, deren Bruttomonatseinkommen dank Boni und Gewinnbeteiligungen um $36 \%$ auf gut $9.300 €$ im Mittel gestiegen ist. 


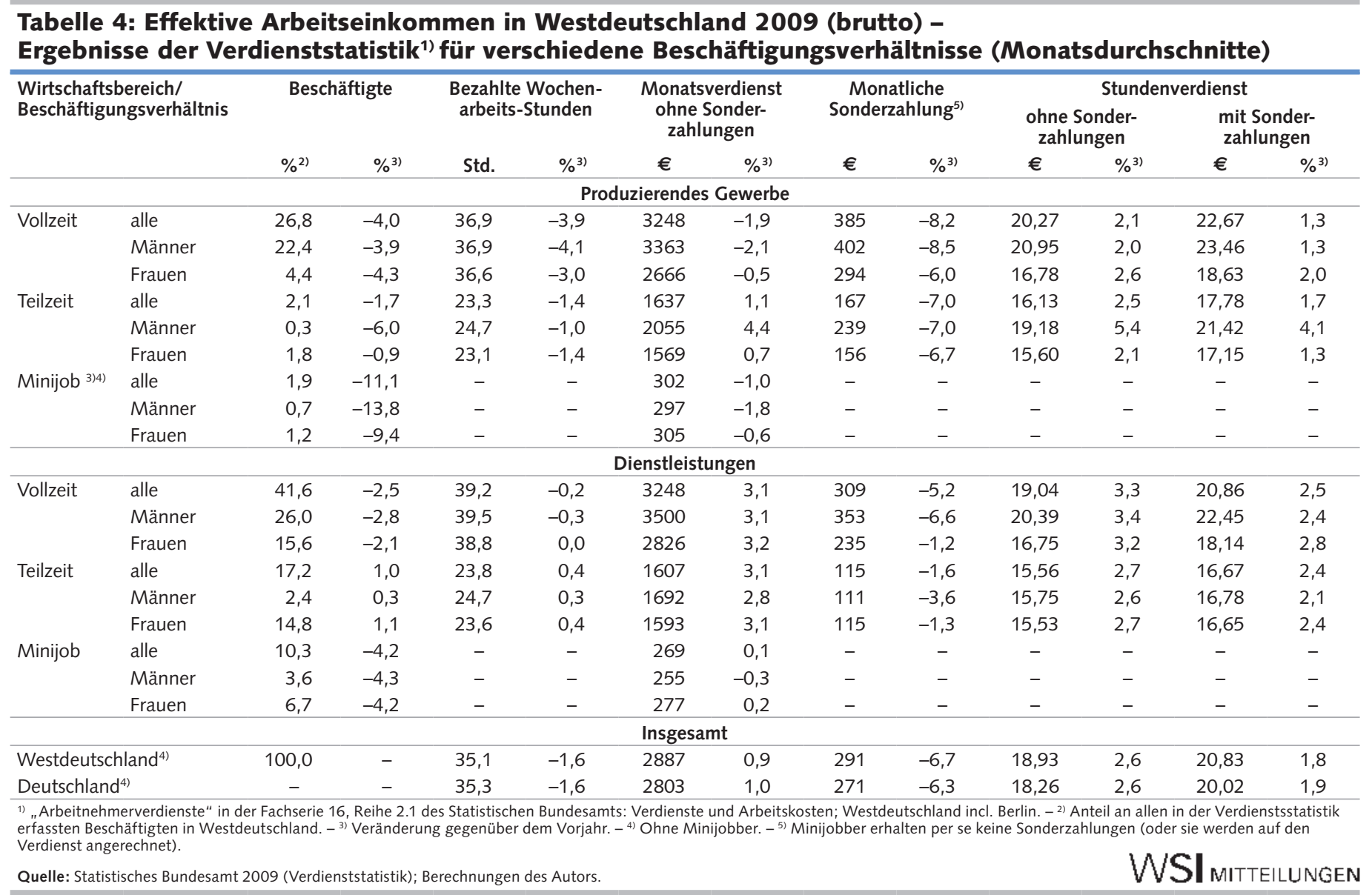

nicht nur von 22,1 Mrd. auf 2,9 Mrd. Euro radikal vermindert; die zuletzt genannte Verlustsumme stammt auch nur aus einer „vergleichsweise geringen Zahl größerer Institute" (Deutsche Bundesbank 2010, S. 17). Die anderen Banken konnten dagegen „zum Teil deutlich gestiegene Jahresüberschüsse vor Steuern aufweisen“" (ebd.). Staatliche direkte und indirekte Hilfen werden daran einen wesentlichen Anteil haben.

$\mathrm{Zu}$ den in der Öffentlichkeit unterschätzten indirekten Hilfen gehört: Die Europäische Zentralbank (EZB) leiht den Banken - und nur diesen und nicht etwa den unter Finanzschwierigkeiten „stöhnenden" EU-Staaten - nach wie vor fast zum Null-Tarif Geld, genauer: zu einem aktuellen Leitzins von $1 \%$. Die als Sicherheiten hinterlegten Staatsanleihen der krisengeschüttelten Mittelmeerländer in der europäischen Union mit ihren hohen „Rating-bedingten“ Marktzinsen haben einen sehr großen Anteil am Anlagenbestand der deutschen und europäischen Banken und bringen deshalb viel Rendite - während die Kosten über den Staat bzw. dessen Steuerlasten und Sparmaßnahmen beim Bürger ankommen (so auch DGB-Bundesvorstand 2010). Private und öffentliche Kreditnehmer werden von den Banken nicht angemessen am niedrigen EZB-Leitzins beteiligt bzw. müssen weiter relativ hohe Zinsen zahlen, bei sogenannten Dispositions-Krediten der Privaten sogar nach wie vor zweistellige. Auch solche Umstände verlangen eigentlich nach einer „ordentlichen“ Bankenabgabe, die eben nicht nur die direkt vom Staat unterstützten Banken betrifft und einen "gesellschaftlichen“ Ausgleich für die vielen kleinen und großen Schutzschirme erbringt.

\section{GEWINNE VON PRODUKTIONS- UNTERNEHMEN}

Eine eigenständige Untersuchung zur Gewinn- und Vermögenssituation der Produktionsunternehmen in Deutschland für 2009 hat die Deutsche Bundesbank bisher nicht vorgelegt. Sie schreibt aber in der entsprechenden Analyse für 2008, die dort Anfang 2010 in Kenntnis der Krise veröffentlicht wurde, die folgenden „beruhigenden“ Sätze. „Trotz des geringeren Jahresergebnisses nahmen die einbehaltenen Gewinne [2008, C.S.] weiter beträchtlich zu. In der Folge verbesserten sich die Innenfinanzierungsmöglichkeiten der Unternehmen erneut; das Eigenkapital erhöhte sich kräftig auf 251/2\% der Bilanzsumme. Die sich im Herbst 2008 zuspitzende Finanz- und Wirtschaftskrise hat sich alles in allem in den untersuchten Jahresabschlüssen deutscher Unternehmen erst wenig bemerkbar gemacht. Im Jahr 2009 dürften die Belastungen aber erheblich zugenommen haben. Die Analyse der Erfolgs- und Bilanzdaten 2008 verdeutlicht jedoch, dass der nichtfinanzielle Unternehmenssektor mit einem im Vergleich zu früheren Schwächephasen höheren Cashflow, einer günstigeren Ertragslage sowie einer merklich robusteren finanziellen Bestandsfestigkeit in den konjunkturellen Abschwung eingetreten ist" (Deutsche Bundesbank 2010b, S. 15).

Indirekt bestätigt die Deutsche Bundesbank (2010c, 2010d) diese Einschätzung durch kurze Veröffentlichungen zur gesamtwirtschaftlichen Finanzierungsrechnung für 2009 und das 1. Quartal 2010 und darin enthaltene Informationen zu den Produktionsunternehmen. Deren Geldver- 
Tabelle 5: Effektive Arbeitseinkommen in Ostdeutschland 2009 (brutto) Ergebnisse der Verdienststatistik ${ }^{1)}$ für verschiedene Beschäftigungsverhältnisse (Monatsdurchschnitte)

\begin{tabular}{|c|c|c|c|c|c|c|c|c|c|c|c|c|c|c|c|}
\hline \multirow{2}{*}{\multicolumn{2}{|c|}{$\begin{array}{l}\text { Wirtschaftsbereich/ } \\
\text { Beschäftigungsverhältnis }\end{array}$}} & \multirow{2}{*}{\multicolumn{2}{|c|}{ Beschäftigte }} & \multirow{2}{*}{\multicolumn{2}{|c|}{$\begin{array}{c}\text { Bezahlte } \\
\text { Wochenarbeits- } \\
\text { Stunden }\end{array}$}} & \multirow{2}{*}{\multicolumn{2}{|c|}{$\begin{array}{c}\text { Monatsver- } \\
\text { dienst ohne } \\
\text { Sonderzahlungen }\end{array}$}} & \multirow{2}{*}{\multicolumn{2}{|c|}{$\begin{array}{l}\text { Monatliche } \\
\text { Sonderzahlung5) }\end{array}$}} & \multicolumn{6}{|c|}{ Stundenverdienst } \\
\hline & & & & & & & & & & \multicolumn{3}{|c|}{ ohne Sonderzahlung } & \multicolumn{3}{|c|}{ mit Sonderzahlung } \\
\hline & & $\%^{2)}$ & $\%^{3)}$ & Std. & $\%^{3)}$ & $€$ & $\%^{3)}$ & $€$ & $\%^{3)}$ & $€$ & $\%^{3)}$ & $\%^{6)}$ & $€$ & $\%^{3)}$ & $\%^{6)}$ \\
\hline \multicolumn{16}{|c|}{ Produzierendes Gewerbe } \\
\hline \multirow[t]{3}{*}{ Vollzeit } & alle & 27,5 & $-3,2$ & 38,3 & $-3,2$ & 2262 & $-0,8$ & 166 & $-4,7$ & 13,59 & 2,5 & 67,0 & 14,59 & 2,2 & 64,4 \\
\hline & Männer & 21,7 & $-3,1$ & 38,3 & $-3,4$ & 2347 & $-0,8$ & 173 & $-4,6$ & 14,10 & 2,6 & 67,3 & 15,14 & 2,4 & 64,5 \\
\hline & Frauen & 5,8 & $-3,6$ & 38,3 & $-2,6$ & 1944 & $-0,8$ & 139 & $-5,6$ & 11,67 & 1,9 & 69,5 & 12,51 & 1,6 & 67,1 \\
\hline \multirow[t]{3}{*}{ Teilzeit } & alle & 1,5 & 0,6 & 28,5 & $-1,8$ & 1256 & 1,7 & 65 & 3,8 & 10,13 & 3,5 & 62,8 & 10,65 & 3,6 & 59,9 \\
\hline & Männer & 0,3 & 7,1 & 27,8 & $-0,8$ & 1634 & 6,2 & 107 & 11,8 & 13,54 & 7,0 & 70,6 & 14,42 & 7,4 & 67,3 \\
\hline & Frauen & 1,3 & $-0,7$ & 28,7 & $-1,9$ & 1172 & 0,0 & 55 & $-0,2$ & 9,40 & 1,9 & 60,3 & 9,85 & 1,9 & 57,4 \\
\hline \multirow[t]{3}{*}{ Minijob 3)4) } & alle & 1,2 & 1,1 & - & - & 239 & $-8,0$ & - & - & - & - & - & - & - & - \\
\hline & Männer & 0,5 & $-3,2$ & - & - & 254 & $-2,5$ & - & - & - & - & - & - & - & - \\
\hline & Frauen & 0,7 & 4,9 & - & - & 227 & $-12,3$ & - & - & - & - & - & - & - & - \\
\hline \multicolumn{16}{|c|}{ Dienstleistungen } \\
\hline \multirow[t]{3}{*}{ Vollzeit } & alle & 43,4 & $-2,8$ & 39,5 & $-0,1$ & 2629 & 4,0 & 169 & 2,1 & 15,32 & 4,2 & 80,5 & 16,30 & 4,1 & 78,1 \\
\hline & Männer & 22,8 & $-3,9$ & 39,8 & $-0,3$ & 2681 & 4,1 & 174 & 3,1 & 15,50 & 4,4 & 76,0 & 16,50 & 4,3 & 73,5 \\
\hline & Frauen & 20,6 & $-1,6$ & 39,1 & 0,0 & 2570 & 3,9 & 164 & 1,0 & 15,11 & 3,9 & 90,2 & 16,08 & 3,8 & 88,6 \\
\hline \multirow[t]{3}{*}{ Teilzeit } & alle & 18,9 & 2,3 & 28,8 & 0,2 & 1650 & 2,8 & 97 & 1,8 & 13,18 & 2,6 & 84,7 & 13,95 & 2,6 & 83,7 \\
\hline & Männer & 2,4 & 5,3 & 28,9 & 0,3 & 1649 & 2,1 & 89 & 1,8 & 13,15 & 1,8 & 83,5 & 13,86 & 1,8 & 82,6 \\
\hline & Frauen & 16,5 & 1,9 & 28,8 & 0,2 & 1650 & 2,9 & 98 & 1,9 & 13,18 & 2,8 & 84,9 & 13,97 & 2,7 & 83,9 \\
\hline \multirow[t]{3}{*}{ Minijob } & alle & 7,5 & $-1,5$ & - & - & 195 & $-0,5$ & - & - & - & - & - & - & - & - \\
\hline & Männer & 3,0 & 0,8 & - & - & 188 & $-1,5$ & - & - & - & - & - & - & - & - \\
\hline & Frauen & 4,5 & $-3,0$ & - & - & 199 & 0,2 & - & - & - & - & - & - & - & - \\
\hline \multicolumn{16}{|c|}{ Insgesamt } \\
\hline \multicolumn{2}{|c|}{ Ostdeutschland ${ }^{4)}$} & 100 & - & 36,7 & $-1,3$ & 2293 & 2,0 & 152 & $-0,7$ & 14,36 & 3,4 & 75,9 & 15,31 & 3,2 & 73,5 \\
\hline \multicolumn{2}{|c|}{ Deutschland ${ }^{4)}$} & - & - & 35,3 & $-1,6$ & 2803 & 1,0 & 271 & $-6,3$ & 18,26 & 2,6 & - & 20,02 & 1,9 & - \\
\hline \multicolumn{16}{|c|}{$\begin{array}{l}\text { 1) "Arbeitnehmerverdienste" in der Fachserie 16, Reihe } 2.1 \text { des Statistischen Bundesamts: Verdienste und Arbeitskosten; Ostdeutschland ohne Berlin. }-{ }^{2)} \text { Anteil an allen in der Verdienstsstatis- } \\
\text { tike erfassten Beschäftigten in Ostdeutschland. }{ }^{3)} \text { Veränderung gegenüber dem Vorjahr. }-{ }^{4)} \text { Ohne Minijobber. }-{ }^{5} \text { Minijobber erhalten per se keine Sonderzahlungen (oder sie werden auf den } \\
\text { Verdienst angerechnet). }{ }^{6)} \text { Relation des ostdeutschen Verdienstniveaus zum jeweiligen westdeutschen Niveau in \%. }\end{array}$} \\
\hline \multicolumn{16}{|c|}{ Quelle: Statistisches Bundesamt 2009 (Verdienststatistik); Berechnungen des Autors. } \\
\hline
\end{tabular}

mögenszuwachs fiel danach Anfang 2010 mit plus 6,5 Mrd. $€$ zwar „vergleichsweise schwach" aus. Doch daneben gab es durchaus beachtlichen anderen Vermögenszuwachs in Form von mehr Investitionen, neuen Firmenbeteiligungen (28 Mrd. €) und Rentenwerten (16,5 Mrd. €) sowie Vergabe von Krediten (8 Mrd. $€$ ). Auch die Außenfinanzierung durch das Emittieren von Aktien und Schuldverschreibungen konnte um 18 Mrd. $€$ gestärkt werden.

Diese Informationen unterstreichen die aus der VGR eingangs genannte sehr positive Entwicklung seit Beginn 2010 bei den Einkommen aus Gewinnen und Vermögen. Der dort zitierte Zuwachs von 19,5\% im ersten Halbjahr 2010 dürfte in dieser Größenordnung auch für das ganze Jahr gelten und angesichts zurzeit niedriger Verzinsung für Vermögensanlagen überwiegend den Unternehmen zufließen. Aktienanalysten rechnen z.B. laut Frankfurter Rundschau (2010b) für die 30 DaxKonzerne mit einer Zunahme ihres Rohgewinns um mehr als $50 \%$ und für die 50 kleineren Unternehmen im M-Dax sogar um $150 \%$.

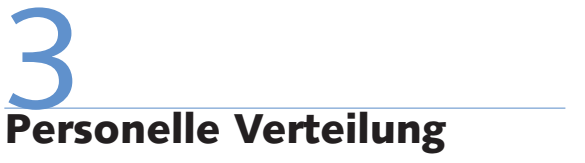

Diese Entwicklungen passen in das Bild, das die erste und bislang einzige Untersuchung der Einkommensverteilung auf der Ebene der privaten Haushalte für das Krisenjahr 2009 mit Hilfe des Sozio-ökonomischen Panels (SOEP) zeichnet. Diese Analyse wurde vom Deutschen Institut für Wirtschaftsforschung (DIW) durchgeführt (Goebel et al. 2010). Danach setzt sich auch im Vorjahr die früher schon auf SOEP-Basis von Grabka/Frick (2008) beobachtete Polarisierung der verfügbaren Einkommen zwischen unten und oben bei gleichzeitigem Schrumpfen der Mittelschicht fort. Und es scheint sich auch die frühere These zu bestätigen, dass eine solche Polarisierungstendenz sich immer mehr loslöst von der tatsächlichen konjunkturellen Entwicklung. Gemessen an den durchschnittlichen Einkommensabständen zwischen den vom DIW gebildeten drei Einkommensgruppen „niedrig“, „mittel“ und „hoch“ hat sich sowohl die sogenannte absolute als auch die sogenannte relative Polarisierung bereits seit 1993 vollzogen (Abbildung 1). „Die Dynamik der Entwicklung hat sich dabei seit 2000 spürbar erhöht. So stieg der absolute Rückstand der unteren zur mittleren Einkommensgruppe von rund $590 € \mathrm{im} \mathrm{Jahr}$ 1999 auf rund $630 € 2009$. Im Verhältnis zu den mittleren Einkommen wuchs der relative Einkommensrückstand von $46 \%$ auf mehr als $48 \%$. Das heißt nichts anderes, als dass die Ärmeren nicht nur immer mehr geworden sind, sondern im Durchschnitt auch immer ärmer werden. Spiegelverkehrtes gilt im Trend auch für die Einkommensdifferenzen der oberen Einkommensgruppe. Der absolute Vorsprung der oberen zur mittleren Einkommensgruppe stieg von $1.165 €$ im Jahr 1999 auf $1.360 € 2009$. Im Verhältnis zu den mittleren Einkommen wuchs der relative Einkommensvorsprung von $91 \%$ auf fast $104 \%$ [...] Auch von 2008 auf 2009 stieg der absolute Vorsprung der oberen zur mittleren Einkommensgruppe nochmals spürbar" (Goebel et al. 2010, S. 5). 
Laut DIW nahm der Anteil der Haushalte mit hohem Einkommen an allen Haushalten 2009 zwar leicht ab. Doch möglicherweise muss diese Aussage wie auch die zum gestiegenen Einkommen dieser Gruppe nachträglich sogar noch zugunsten von „oben“ korrigiert werden. Denn die aktuelle DIW-Untersuchung kann sich nur auf die direkt im SOEP abgefragten laufenden verfügbaren Monatseinkommen stützen. Die noch aussagefähigeren Jahreseinkommen der privaten Haushalte, die auch unregelmäßige Einkommenszuflüsse wie z.B. Boni, Zinsen und andere Vermögenserträge enthalten, werden vom DIW auf Basis aller verfügbaren Befragungsinformationen und nachfolgender aufwendiger Bereinigungsprozesse erst etliche Zeit später ausgewertet. Nach Erfahrungen aus diversen Befragungen fallen dabei aber bei hohen Einkommen die zusätzlichen Einkünfte stärker aus als bei mittleren und erst recht unteren Einkommen, weil Erstere nicht zuletzt aufgrund ihrer vorhandenen Vermögen über mehr und auch über ergiebigere Einkommensquellen verfügen.

Außerdem ist in diesem Zusammenhang immer wieder auf die in freiwilligen Befragungen begrenzte Erfassbarkeit gerade sehr hoher Einkommen hinzuweisen. Das gilt für das SOEP wie auch für die Einkommen- und Verbrauchsstichprobe (EVS), die beide verfügbare Monatseinkommen oberhalb von rund $20.000 €$ nicht ausreichend darstellen können. D.h. die Spitze der Einkommen (und Vermögen) in Deutschland kommt in den erwähnten Daten ohnehin nicht zum Ausdruck. Diese werden allenfalls auf Basis von Unternehmens-, Bank- und Börseninformationen gelegentlich geschätzt wie zuletzt wieder vom Manager-Magazin (2010) bezüglich der 500 reichsten Personen und Familien in Deutschland. Danach hat z.B. die Zahl der Milliardäre 2009 trotz Krise sogar um vier Personen auf 103 Personen zugenommen. Und das - erheblich von volatilen Aktienkursen geprägte - Vermögen dieser Reichen-Gruppe ist 2009 immerhin insgesamt um $0,5 \%$ gestiegen. Auf internationaler Ebene belegen Schätzungen zu hohen Einkommen und großen Vermögen wie z.B. der Global Wealth Report 2010 der Unternehmensberatung Boston Consulting Group (Becerra et al. 2010), dass der „weltweite“ "Reichtum“ 2009 sogar schon wieder über dem Niveau der 2008 ausgebrochenen Finanz- und Wirtschaftskrise lag. Der Trend ist ersichtlich, auch wenn sich hinter solchen aggregierten Zahlen viele individuelle

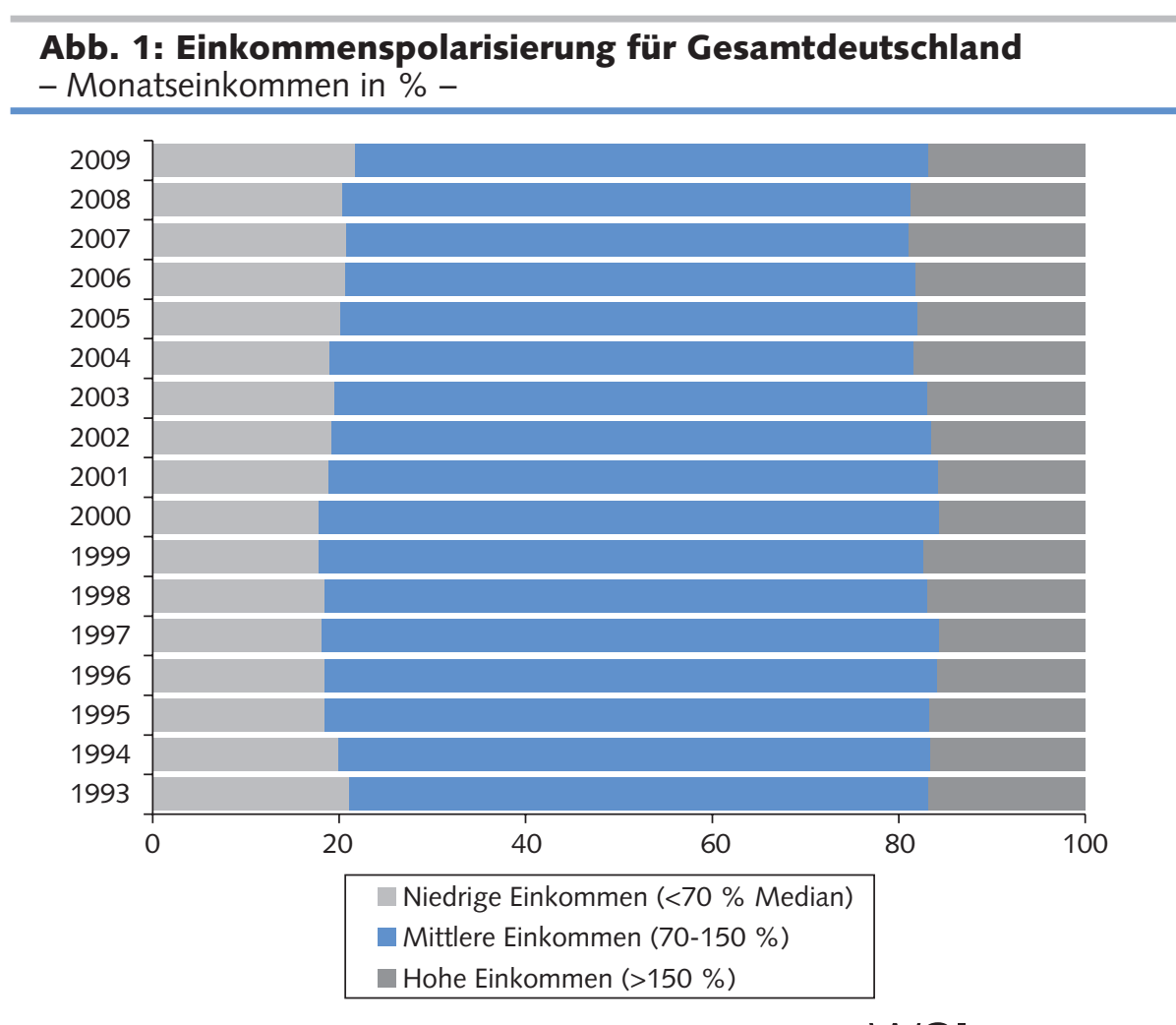

Quelle: SOEP; DIW (2010).

WSI MITTELLUNGEN

Unterschiede und darunter auch Aufstiegsund Abstiegsprozesse - wie in der gesamten Einkommensverteilung - verbergen.

Eindeutiger als die Informationen zum Reichtum sind diejenigen zum anderen Ende der Einkommenspyramide. Auch dazu gibt es vom DIW (für Deutschland) eine aktuelle Studie (Grabka/Frick 2010), die die anhaltende Armutsproblematik hierzulande belegt. Man kann in diesem Zusammenhang nicht genug darauf hinweisen, dass Einkommensarmut in der Regel auch mit individueller Chancenarmut einhergeht (so z.B. Allmendinger 2010; Allmendinger et al. 2010) - und in dieser Kumulation den „doppelten“ Sprengsatz auf gesellschaftlicher und gesamtwirtschaftlicher Ebene bildet.

Die Armuts- und Reichtumsentwicklung in Deutschland findet übrigens seit Jahren eine ähnliche Entsprechung in den anderen EU-Ländern. So konstatiert jüngst Dauderstädt (2010), dass die Einkommensverteilung in der Europäischen Union ,viel ungleicher (ist) als die Europäische Union selbst angibt" - sogar "ungleicher als etwa in Indien" (ebd. S. 1). Zwar habe die erhebliche Ungleichheit seit 2005 - soweit messbar im europäischen Durchschnitt abgenommen; aber bei zunehmender Ungleichheit in den meisten Mitgliedstaaten sei dies auf den relativen Aufholprozess in den ärmeren EULändern zurückzuführen. Letzterer jedoch ist heute durch die Finanzkrise nicht nur jäh gestoppt - er droht wegen der dort eingeschlagenen Sparpolitik und anderer Krisenfolgen auch zurückgedreht zu werden.

\section{1 \\ Verteilung - Wachstum - Zukunft}

\subsection{QUO VADIS DEUTSCHLAND?}

Das wirft die Frage nach der zukünftigen deutschen Entwicklung und der hiesigen Bedeutung der Krise auf. Vor dem Hintergrund der in der Krise noch deutlicher gewordenen Zusammenhänge zwischen ungleicher Verteilung und anderen krisenträchtigen Ungleichgewichten auf nationaler wie internationaler Ebene (vgl. Fitoussi/Stiglitz 2009 oder die bei Schäfer 2010 zitierte Literatur) zeichnet sich ein wenig optimistisch stimmendes Szenario ab. Denn so sehr im Moment die Produktion generell und der Arbeitsmarkt partiell vom exportabhängigen bzw. außengesteuerten Wachstum profitieren: Die weiteren Aussichten bleiben voller Risiken. Und das 
nicht nur wegen diverser sich abzeichnender Weltmarktprobleme, sondern auch wegen der schon vorhandenen Binnenmarktprobleme. Vor allem gehen beide wesentlich auf die ungleiche Einkommensverteilung und das dahinter stehende deutsche „Wachstums-Modell“ zurück, dessen dynamischer Export-Erfolg ${ }^{3}$ mehrfach teuer erkauft worden ist. Das wurde auch in den WSI-Verteilungsberichten der vergangenen Jahre immer wieder thematisiert.

Die jahrelange Umverteilung zulasten von privaten Löhnen und öffentlichen Einnahmen hat zwar den Exportanstieg wesentlich möglich gemacht, aber gleichzeitig die internen Wachstumspotenziale beschnitten, weil sie auf Kosten der privaten und öffentlichen Nachfrage ging, soziale Probleme erzeugte, das private Bildungsvermögen beschädigte, das öffentliche Infrastrukturvermögen vernachlässigte und selbst die privaten Investitionen, sprich: die Erneuerung des Anlagevermögens, nur mäßig anregte. Und das damit bezahlte deutsche Wachstum musste im internationalen Vergleich bescheiden bleiben. Gleichwohl belastete dieses deutsche Wachstum andere Länder. Denn der zunehmende Exporterfolg bedrängte die per Saldo importierenden europäischen Nachbarländer in der Euro-Zone immer mehr, weil dort mit deutschen Waren und Dienstleistungen bei festen Wechselkursen auch Arbeitslosigkeit „importiert“ wurde. Der Preis dafür ist eine drohende Beschädigung von europäischer Wirtschafts-, Währungs- und Politikunion. Und schließlich vergrößerte der kräftig gestiegene Exportüberschuss-Anteil am BIP die Abhängigkeit Deutschlands vom Weltmarkt so sehr, dass deutsche Produktion und Wertschöpfung in der Krise mit am stärksten einbrachen. Wenn jetzt im Zuge einer Erholung der Außennachfrage vor allem aus Asien der deutsche Export noch stärker steigt und zu seiner Absicherung die Umverteilung noch weiter verschärft wird, werden die genannten Probleme erneut virulent, kurz- und mittelfristig vielleicht sogar noch größer. Dabei wäre ein schnelles Ende des nur „geliehenen“ aktuellen Wachstums nur ein Szenario, die fehlende Kompensationsmöglichkeit durch die unterentwickelte deutsche Binnennachfrage lediglich ein zweites.

\subsection{WELTWIRTSCHAFTLICHE RISIKEN}

Vom Weltmarkt drohen nachlassende Wachstumsimpulse, wenn nicht sogar aufziehende neue Krisenfaktoren durch
- die Auswirkungen der „Austeritätspolitik" in immer mehr EU-Ländern (zuletzt in Großbritannien);

- die vom Finanzmarkt erzwungenen neuen Staatsschulden und ihre auf Bürgerkosten erfolgenden Bewältigungsstrategien; - die Bremsspuren in den jetzt noch expandierenden asiatischen Ländern, die teils der Furcht vor eigenen Spekulationsblasen, teils aber der Sättigung ihrer Produktionskapazitäten durch importierte deutsche und ausländische Maschinen und Anlagen entstammen;

- die ausbleibende ökonomische Erholung in den USA, die die Bremsspuren in Asien verstärkt und auch die Exporte aus Europa erneut beschneiden könnte;

- einen von diesen Aussichten motivierten internationalen Wettlauf zur Abwertung bzw. zur Erhöhung der eigenen Exportmächtigkeit über eine nominale Senkung der Währungskurse oder eine reale Senkung der Lohnkosten nach deutschem Vorbild;

- eine mögliche spezifische Gegenwehr der vom deutschen Exportüberschuss belasteten Nachbarländer in der Euro-Zone (so z.B. der luxemburgische Ministerpräsident Juncker (2010)).

Angesichts solcher Gefahren von außen und der jüngst gemachten Krisenerfahrungen läge es mehr als nahe

- erstens, die Binnennachfrage zu stär$\mathrm{ken}^{4}$, die im Gegensatz zum Weltmarkt auf nationaler Ebene steuerbar ist (so z.B. auch IMK-Arbeitskreis Konjunktur 2010; Schrooten/Teichmann 2010; Kromphardt 2010; Arbeitsgruppe Alternative Wirtschaftspolitik 2010; u.a.m.)

- zweitens, Konsequenzen daraus zu ziehen, dass durch die bisherigen Exportüberschüsse nicht nur internationale Ungleichgewichte verstärkt, sondern auch schon nationale Ressourcen „verloren“ gegangen sind, die alternativ in mehr quantitatives wie qualitatives Wachstum und eine nachhaltige Zukunft hierzulande hätten investiert werden können.

Doch das Gegenteil ist der Fall.

\subsection{BINNENWIRTSCHAFTLICHE RISIKEN}

Tat und Plan der aktuellen deutschen Politik veranschaulichen das ausbleibende Lernen aus der Krise. Zwar werden die oberen
Einkommen und Vermögen nicht mehr wie ursprünglich beabsichtigt zusätzlich von Steuern entlastet. Aber sie werden weiter direkt und indirekt "geschont" durch

- die Einführung des ermäßigten Mehrwertsteuersatzes auf Hotelübernachtungen; - die evtl. völlige Befreiung von der Mehrwertsteuer für ausgelagerte Servicegesellschaften von Finanzdienstleistern laut einer Gesetzesinitiative des Bundesrats (Süddeutsche Zeitung 2010b);

- die Ablehnung eines allgemeinen gesetzlichen Mindestlohns, obwohl der neben den Lohnempfängern auch die öffentlichen Kassen erheblich entlasten könnte; ${ }^{5}$ - den Verzicht auf eine zumindest nationale Finanztransaktionssteuer, obwohl die angesichts des deutschen Gewichts in Europa ohne Weiteres möglich wäre und etwa 8 Mrd. $€$ jährlich zugunsten des Fiskus erzielen würde (Schulmeister 2010; Dieter 2010); - die Verlängerung der AKW-Laufzeiten, deren erheblicher Zusatzgewinn für die Betreiber in keinem Verhältnis zu den kläglichen - in der Höhe ohnehin unsicheren - Einnahmen der zukünftigen Kernbrennstoffsteuer steht;

- eine mehr als bescheidene Bankenabgabe; - das geplante Einfrieren der Arbeitgeberbeiträge zur gesetzlichen Krankenversicherung ab 2011 und anderes mehr.

Auch wegen der damit benannten Einnahmeverzichte - die frühere aufgrund diverser Steuerentlastungen verstärken - werden die öffentlichen Haushalte auf einen erneuten Sparkurs getrimmt, der nicht zuletzt wegen der gesetzlichen Schuldenbremse im Bund und immer mehr Bundesländern Jahr für Jahr härter ausfallen soll (kritisch dazu Horn/ Truger 2010). Und schließlich wird die private Binnennachfrage weiter eingeschnürt, insbesondere bei den unteren Einkommen. $\mathrm{Zu}$ den entsprechenden Maßnahmen zählen

3 Zur wachstumstragenden Exportdynamik vgl. ausführlich Joebkes et al. 2010 sowie Stephan/Redle 2010. Dort wird auch eine Behauptung des Instituts der Deutschen Wirtschaft (Grömling/Matthes 2010) widerlegt, dass die Binnennachfrage die treibende Wachstumsursache gewesen sei.

4 Zwar haben im 2. Quartal 2010 die privaten Haushalte laut VGR erstmals seit einem Jahr wieder mehr konsumiert, jedoch ist gerade mal ein Plus von rund 0,6 \% gegenüber dem 1. Quartal 2010 zu verzeichnen (Fichtner et al. 2010).

5 Seit 2005 hat die öffentliche Hand das Aufstocken von Hartz-IV-Leistungen, das erheblich auf zu niedrige Löhne zurückgeht, nach Angaben der Bundesregierung rund $50 \mathrm{Mrd}$. $€$ gekostet. 
- die indiskutabel geringe Erhöhung der Hartz-IV-Regelsätze nur für Erwachsene und das in der Umsetzung unsichere „Bildungspaket" für ihre Kinder (zusammen 620 Millionen $€) ;{ }^{6}$

- die Streichung des Elterngeldes für Hartz-IV-Empfänger (450 Mio. €);

- die Einschränkung von Einmalzahlungen, Wohnkostenzuschüssen und andere Restriktionen bei Hartz-IV-Empfängern; - die Streichung der Heizungskomponente beim Wohngeld;

- die Erhöhung der GKV-Beiträge ab 2011 und die verstärkte Zahlung von Zusatzbeiträgen - wobei Letztere nach dem geplanten Einfrieren der Arbeitgeberbeiträge ab 2011 mehr und mehr den Charakter einer immer größer werdenden zusätzlichen Kopfpauschale annehmen und zudem wegen des anvisierten Sozialausgleichs kleine Einkommen überproportional belasten werden (so z.B. Blank/ Leiber 2010);

- die von Regierungsseite angekündigten Erhöhungen der Beitragssätze in der Arbeitslosenversicherung und wahrscheinlich auch in der Pflegeversicherung;

- die Aussichten auf höhere Mieten durch die geplante gesetzliche Erleichterung der Umlegung von Energiespar-Maßnahmen der Vermieter;

- die absehbaren Stromkostensteigerungen durch fehlsteuernde Subventionen für die Erzeugung erneuerbarer Energien.
Das alles werden vielleicht kommende höhere Nominallöhne schwerlich wettmachen können, auch wenn sie kräftiger steigen sollten als selbst im vergangenen Aufschwung. Zwar gibt es z.Zt. sogar eine „regierungsamtliche “ öffentliche Unterstützung für deutliche Lohnerhöhungen. Aber ob die nicht bloß rhetorisch zur Aufhellung der getrübten politischen Großwetterlage und der Chancen bei baldigen Wahlen gemeint ist, muss sich noch zeigen. Das politische Einschrumpfen einer Initiative der EUKommission zur Bekämpfung aller Ungleichgewichte - auch des deutschen Exportüberschusses - auf die bisher übliche verkürzte Sichtweise zu den angeblich allzu problematischen Defiziten der öffentlichen Haushalte erzeugt jedenfalls wenig Hoffnung auf politische Lernprozesse.

Skeptisch stimmen auch die tarifpolitischen Entwicklungen. So lässt sich erstens aus den Tarifabschlüssen der letzten Zeit ableiten, dass sich Tendenzen zur Verlängerung der Tariflaufzeiten wie zur Verzögerung des Inkrafttretens von Lohnerhöhungen verstärken (Bispinck et al. 2010a). Zweitens nehmen Pauschal- und Einmalzahlungen zu, die den zukünftigen Lohnanstieg erheblich dämpfen (Bispinck et al. 2010b; Statistisches Bundesamt 2010e). Und drittens belaufen sich die 2010 wirksam gewordenen Tariferhöhungen, die ohnehin wegen nachlassender Tarifbindung nur noch rund die Hälfte aller Beschäftigten direkt erreichen, gerade einmal auf durchschnittlich 1,7\% (Bispinck et al. 2010a). Eine solche nominale Erhöhung kann, das lehrt die Vergangenheit, durch steigende Preise und höhere Abgaben sehr schnell zum Minus bei den realen und verfügbaren Einkommen werden. Und auch der Niedriglohnsektor wird nicht etwa eingedämmt: Beispielsweise soll die Praxis von Unternehmen, Beschäftigte formal auszulagern und über eine unternehmenseigene Verleihfirma zu niedrigeren Lohntarifen zurückzuholen, legalisiert werden. Und ein Mindestlohn in der Arbeitnehmer-Verleihbranche wird trotz der vollen Arbeitnehmer-Freizügigkeit in der EU ab 1.5.2011 selbst auf Branchenebene nicht hergestellt.

Angesichts solcher Vorzeichen und Risiken ist der vielerorts vorhandene Konjunktur-Optimismus (prominent hierfür: Projektgruppe Gemeinschaftsdiagnose 2010) nicht nachvollziehbar (hierzu zugespitzt: Flassbeck 2010). Doch noch mehr Unverständnis verdient die anhaltende Orientierung von Politik und Wirtschaft am „alten“ Modell des Export-Weltmeisters. Dessen Fortsetzung negiert die Vergangenheitserfahrungen und birgt erhebliche Zukunftsrisiken.

\footnotetext{
6 Im "Gegenzug " wurden zugunsten von mittleren und hohen Einkommen seit Anfang 2010 noch 4,6 Mrd. $€$ durch mehr Kindergeld und höhere steuerliche Kinderfreibeträge ausgegeben.
}

\section{LITERATUR}

Allmendinger, J. (2010): Für die Zukunft nicht gewappnet. Ergebnisse der empirischen Studie „Kaum Bewegung, viel Ungleichheit“ im Auftrag der Heinrich-Böll-Stiftung, in: Böll 3, Berlin, S. 6-8

Allmendinger, J./Ebner, Ch./Nikolai, R. (2010): Bildung in Europa 2010 - Ziele erreicht oder verfehlt?, in: WSI-Mitteilungen 4, S. 171-178

Arbeitsgruppe Alternative Wirtschaftspolitik (2010a): Deutsche Zweiheit - Oder: Wie viel Unterschied verträgt die Einheit? Bilanz der Vereinigungspolitik, Köln

Arbeitsgruppe Alternative Wirtschaftspolitik (2010b): Politik hat nichts gelernt - das Umverteilen von unten nach oben geht weiter, Sondermemorandum 2010, Bremen

Becerra, J./Damisch, P./Holley, B./Kumar, M./Naumann, M./Tang, T./Zakrzewski, A. (2010): Regaining Lost Ground - Resurgent Markets and New Opportunities, Report Global Wealth 2010, Boston

Bispinck, R./Wirtschafts- und Sozialwissenschaftliches Institut in der Hans-Böckler-Stiftung (WSI) Tarifarchiv (2010a): Tarifpolitischer Halbjahresbericht - eine Zwischenbilanz der Lohn- und Gehaltsrunde 2010, Informationen zur Tarifpolitik des WSI, Juli 2010, Düsseldorf
Bispinck, R./Wirtschafts- und Sozialwissenschaftliches Institut in der Hans-Böckler-Stiftung (WSI) Tarifarchiv (2010b): Tarifliche Pauschalund Einmalzahlungen. Praxis, Risiken und Nebenwirkungen, Elemente qualitativer Tarifpolitik 69, Düsseldorf

Blank, F./Leiber, S. (2010): Nachhaltige Finanzierung des Gesundheitssystems ohne Kopfpauschale, in: WSI-Mitteilungen 10, S. 542-543 Castel, R./Dörre, K. (Hrsg.) ( 2009): Prekarität, Abstieg, Ausgrenzung. Die soziale Frage am Beginn des 21. Jahrhunderts, Frankfurt a.M./New York

Dauderstädt, M. (2010): Europas unterschätzte Ungleichheit, in: WISO direkt, Friedrich Ebert Stiftung, September, Bonn

Deutsche Bundesbank (2010a): Die Ertragslage der deutschen Kreditinstitute im Jahr 2009, in: Monatsbericht 9, Frankfurt a.M., S. 17-48

Deutsche Bundesbank (2010b): Ertragslage und Finanzierungsverhältnisse deutscher Unternehmen im Jahr 2008, in: Monatsbericht 1, Frankfurt a.M., S. 15-30

Deutsche Bundesbank (2010c): Vermögensbildung und Finanzierung im Jahr 2009, Pressenotiz vom 29.4., Frankfurt a.M. 
Deutsche Bundesbank (2010d): Sektorale Vermögensbildung und Finanzierung im ersten Quartal 2010, Pressenotiz vom 28.7., Frankfurt a.M. DGB-Bundesvorstand (2010): Banken machen Milliarden zum Nulltarif, in: DGB-Klartext vom 20.9., Berlin

Dieter, H. (2010): Chancen und Effekte der Besteuerung von Finanzmarkttransaktionen, Studie der Stiftung Wissenschaft und Politik 24, Berlin

Eichhorst, W./Marx, P./Thode, E. (2010): Atypische Beschäftigung und Niedriglohnarbeit, Bericht auf Basis eines Projekts im Auftrag der Bertelsmann Stiftung, IZA Research Report 25, Bonn

Fichtner, F./Bernoth, K./Bremus, F./Brenke, K./Dreger, Ch./Große Steffen, Ch./Hagedorn, H./Kuzin, V./Pijnenburg, K./Podstawski, M./ Schlitzer, Ch., (2010): Herbstgrundlinien 2010 (Die Herbstprognose des DIW), in: DIW Wochenbericht 39, Berlin, S. 2-16

Fitoussi, J.-P./Stiglitz, J. E. (2009): The Ways Out of the Crisis and the Building of a More Cohesive World, OFCE Document de travail 17, Paris Flassbeck, H. (2010): Zum "Wahnsinn der Institute" in der so genannten Gemeinschaftsdiagnose Herbst 2010, in: NachDenkSeiten vom 15.10., www.nachdenkseiten.de/wp-print.php?p=7106 (letzter Zugriff: 2.1.2010)

Frankfurter Rundschau (2010a): Löhne - Bank-Boni als Motor, Ausgabe vom 22.9., S. 18

Frankfurter Rundschau (2010b): Sie schwimmen schon wieder in Milliarden - Gewinne der Unternehmen explodieren, Ausgabe vom 25.8., S. 14 Garz, M. (2010): The NAIRU and the Extent of the Low-Pay-Sector, IMK Working Paper 12, Düsseldorf

Gerlinger, Th. (2010): Die finanzielle Belastung der Versicherten in der gesetzlichen Krankenversicherung. Vortrag vor der Reformkommission "Für ein solidarisches Gesundheitssystem der Zukunft“ von DGB und Sozialverbänden vom 21.4., Berlin

Goebel, J./Gorning, M./Häußermann, H. (2010): Polarisierung der Einkommen: Die Mittelschicht verliert, in: DIW Wochenbericht 24, S. 2-8 Grabka, M.M./Frick, J. R. (2008): Schrumpfende Mittelschicht - Anzeichen einer dauerhaften Polarisierung der verfügbaren Einkommen?, in: DIW-Wochenbericht 10, S. 101-108

Grabka, M.M./Frick, J. R. (2010): Weiterhin hohes Armutsrisiko in Deutschland: Kinder und junge Erwachsene sind besonders betroffen, in: DIW Wochenbericht 7, S. 2-11

Greß, S./Jacobs, K./Schulze, S. (2010): GKV-Finanzierungsreform: Schwarz-gelbe Irrwege statt gezielter Problemlösungen, in: Gesundheits- und Sozialpolitik 4, S. 14-27

Grömling, M./Matthes, J. (2010): Wachstumsbeiträge auf Branchenebene, IW-Trends 3, Köln

Horn, G./Truger, A. (2010): Haushalts-Begleitgesetz 2011: Die falsche Antwort auf die finanz- und steuerpolitischen Herausforderungen, IMK Policy Brief, Oktober, Düsseldorf

IMK-Arbeitskreis Konjunktur (2010): Erholung verlangsamt sich Prognose updates: Deutsche Konjunktur im Herbst 2010, IMK-Report 55, Oktober, Düsseldorf

Joebges, H./Lindner, F./Nichoj, T. (2010): Mit dem Export aus der Krise? Deutschland im Euroraumvergleich, IMK Report 53, August,

Düsseldorf

Juncker, J.-C. (2010): Kritik an der Exportnation - Juncker wirft

Deutschland "Sozialdumping" vor. Der luxemburgische Premierminister im Interview mit der Zeitung Das Wort vom 11.08., Luxemburg, http://www.wort.lu/wort/web/letzebuerg/artikel/2010/08/107883/ juncker-wirft-deutschland-sozialdumping-vor.php (letzter Zugriff: 2.11.2010)
Kalina, T./Weinkopf, C. (2010): Niedriglohnbeschäftigung 2008: Stagnation auf hohem Niveau - Lohnspektrum franst nach unten aus, IAQ-Report 6, Duisburg

Kienbaum-Managementberatung (2010): Vergütungsreport Leitende Angestellte 2010, Gummersbach

Kromphardt, J. (2010): Sparpaket: Weder gut für Deutschland noch für unsere Nachbarn, in: Wirtschaftsdienst 7, Hamburg, S. 426-427

Kuhner, Ch./Hitz, J.-M./Sabiwalsky, R. (2010): Studie Managergehälter 2010. Vorstandsvergütung deutscher börsennotierter Aktiengesellschaften: Analyse-Entwicklung-Transparenz, Köln

Ludwig, U. (2010): Arbeitskosteneffekte des Vorleistungsbezugs der Industrie an Dienstleistungen in Deutschland im Vergleich zu Frankreich und den Niederlanden, Untersuchung im Auftrag des IMK, IMK-Study 4, Halle/Düsseldorf

Manager-Magazin (2010): Die 500 reichsten Deutschen, Sonderausgabe des Manager-Magazins vom 12.10.

Projektgruppe Gemeinschaftsdiagnose (2010): Gemeinschaftsdiagnose Herbst 2010. Deutschland im Aufschwung - Wirtschaftspolitik vor wichtigen Entscheidungen, München

Schäfer, C. (2010): Die Krise ist nur eine Spitze des Eisbergs, in: Heimbach-Steins, M. (Hrsg.): Weltwirtschaft und Gemeinwohl - Eine Zwischenbilanz der Wirtschaftskrise, Jahrbuch für Christliche Sozialwissenschaften 51, Münster, S. 65-80

Schrooten, M. /Teichmann, I. (2010): Export wieder auf Touren - Binnennachfrage muss nachziehen, in: DIW Wochenbericht 35, S. 2-8 Schulmeister, St. (2010): Notfalls (die Finanztransaktionssteuer) im Alleingang, in: Süddeutsche Zeitung vom 11.10., S. 18

Statistisches Bundesamt (2010a): Inlandsproduktberechnung, Stand August 2010, Fachserie 18, Reihen 1.2 (Vierteljahresergebnisse) und 1.4 (Jahresergebnisse), Wiesbaden

Statistisches Bundesamt (2010b): Verdienste und Arbeitskosten - Arbeitnehmerverdienste 2009, Fachserie 16, Reihe 2.3, Wiesbaden Statistisches Bundesamt (2010c): Arbeitskosten im 2. Quartal 2010: $+0,4 \%$ gegenüber Vorquartal, Pressemitteilung 311 vom 08.09., Wiesbaden

Statistisches Bundesamt (2010d): 2. Quartal 2010: Reallöhne steigen um 2,3\%, Pressemitteilung 335 vom 22.09., Wiesbaden

Statistisches Bundesamt (2010e): Tarifabschlüsse im ersten Halbjahr 2010 sahen häufig Einmalzahlungen vor, Pressemitteilung 317 vom 09.09., Wiesbaden

Stephan, S./Redle, L. (2010): Going East - Deutschland setzt auf Handel mit China und den Ländern Mittel- und Osteuropas, IMK Report 54, September, Düsseldorf

Süddeutsche Zeitung (2010a): Ärger über Banker-Gehälter, Ausgabe vom 27.9.

Süddeutsche Zeitung (2010b): Verdi fürchtet Lohndumping bei Banken, Ausgabe vom 18.10.

Wage Indicator Foundation (Hrsg.) (2010): Income gap starters - A comparative analysis of starter's earnings in eleven occupations and fourteen countries, based on the Wage Indicator dataset, Oktober, www.wageindicator.org 